\title{
What Does the Incompleteness Theorem Add to the Unsolvability of the Halting Problem?
}

\author{
Torkel Franzén ${ }^{\dagger}$ \\ Systemteknik, LTU, SE-971 87 Luleå, Sweden \\ torkel@sm.luth.se
}

Turing's paper including a proof of the unsolvability of the halting problem appeared five years after Gödel's 1931 paper, and ever since there have been many and close ties between uncomputability and incompleteness. The purpose of my talk is to discuss the specific role and contribution of the "Gödelian" approach to incompleteness.

† Torkel Franzén died on April 19, 2006. 